REGARDS

SUR LEECONOMIE ALLEMAND

BULLETIN ECONOMIQUE DU CIRAC
Regards sur l'économie allemande

Bulletin économique du CIRAC

$88 \mid 2008$

Varia

\title{
Les vraies causes de la disparité des revenus en
}

RFA

Stefanie Wahl

Traducteur : Isabelle Bourgeois

\section{OpenEdition}

Journals

Édition électronique

URL : http://journals.openedition.org/rea/1433

DOI : 10.4000/rea. 1433

ISBN : 978-2-8218-0872-0

ISSN : 1965-0787

Éditeur

CIRAC

Édition imprimée

Date de publication : 1 octobre 2008

Pagination : 27-34

ISSN : 1156-8992

\section{Référence électronique}

Stefanie Wahl, «Les vraies causes de la disparité des revenus en RFA », Regards sur l'économie allemande [En ligne], 88 | octobre 2008, mis en ligne le 01 octobre 2010, consulté le 20 avril 2019. URL : http://journals.openedition.org/rea/1433 ; DOI : 10.4000/rea.1433 


\title{
Les vraies causes de la disparité des revenus en RFA
}

\author{
Stefanie Wahl
}

Les études et rapports sur la disparité croissante des revenus fleurissent outreRhin en cette longue période électorale qui s'étend des élections au Landtag de Hesse le 27 janvier 2008 (dont la constitution du gouvernement a été retardée jusqu'ici, les partis établis se refusant à sceller une coalition avec la nouvelle formation marxiste Die Linke) jusqu'aux élections au Bundestag, programmées le 27 septembre 2009. Dans ce contexte, les débats se focalisent d'une part sur la contraction de cette classe moyenne qui porte les valeurs "bourgeoises " au centre du modèle économique et social allemand (voir REA 84/07) et qui se sent gagnée aujourd'hui par un profond malaise. Comme en France, elle voit son pouvoir d'achat en berne face à l'inflation, et est victime elle aussi en partie d'une perception en porte-à-faux entre la hausse globale des revenus et son "vouloir d'achat " (Moat/Rochefort, 2008). Mais alors qu'en France, le débat reste centré dans l'ensemble sur la question générale du pouvoir d'achat, outre-Rhin, il porte sur le risque de déclassement et, surtout, de pauvreté frappant de plus en plus d'Allemands (voir REA 87/08). Cette concentration thématique obéissant à une stratégie politique dont l'objectif est de forcer l'adoption d'un SMIC légal généralisé, les analyses de l'évolution globale des revenus comme de la nette extension de la catégorie des faibles revenus sont rarement objectives, bien qu'elles se nourrissent la plupart du temps de données chiffrées incontestables, car scientifiquement établies. C'est en effet l'interprétation des statistiques qui est faussée par les intentions des acteurs du débat.

Dans ce contexte, l'Institut für Wirtschaft und Gesellschaft (IWG, Bonn), avait publié au début de l'été une étude sur "Les gagnants et les perdants " de l'évolution des revenus au cours des dix dernières années. Elle propose une lecture structurelle, sociodémographique, des disparités croissantes observées, et apporte ainsi un peu plus d'objectivité et de sérénité dans le débat. Nous avons demandé à Stefanie Wahl, qui en est l'un des auteurs, de nous en exposer les principales conclusions. (IB)

\section{Profond malaise des classes moyennes}

Au cours des deux dernières années, l'économie allemande a connu une croissance cumulée de plus de $5 \%$, le PIB a augmenté de 126 milliards $€$ aux prix actuels, et 1,5 million d'emplois ont été créés. Et pourtant, malgré cette évolution positive, les Allemands sont d'humeur maussade, préoccupés par la peur du déclassement social. A en croire par exemple un sondage réalisé en mai 2008 par l'institut ipos (Mannheim) pour le compte de la Fédération des banques allemandes, la moitié seulement $(55 \%)$ des personnes interrogées pense vivre dans une société prospère ; voici deux ans, ce taux était encore de $65 \%$. Aujourd'hui, $40 \%$ des sondés ne voient même aucune prospérité ; en 2006, les pessimistes n'étaient encore que $29 \%$. La mélancolie des Allemands s'explique par l'impression qu'ils ont de ne plus prendre part à la hausse du niveau matériel de la prospérité, ainsi que le révèle par exemple l'édition de juillet du baromètre $A R D$ Deutschlandtrend réalisé par l'institut infratest-dimap pour la première chaîne publique allemande : les trois quarts de la population affirment ne pas profiter de la croissance.

Les Allemands pensent ne pas profiter de la croissance... 
... et craignent pour leur pouvoir d'achat

Les classes moyennes redoutent un déclassement

La répartition des revenus est ressentie comme injuste...

... surtout dans les catégories moyennes et supérieures

Perte de confiance dans l'économie sociale de marché

De plus fortes attentes envers les pouvoirs publics
Ce pessimisme se nourrit de la forte hausse des prix de la plupart des biens essentiels, alors que les salaires et les pensions de retraite n'augmentent pas proportionnellement à l'inflation. Les Allemands aussi ont peur pour leur pouvoir d'achat : l'inflation inquiète au plus haut point $85 \%$ des Allemands, à en croire le même baromètre. Et elle les amène à envisager l'avenir avec la plus vive inquiétude : $46 \%$ d'entre eux pensent que leur situation matérielle se sera détériorée dans dix ans ; ce taux atteint même $52 \%$ chez les plus de 60 ans.

Depuis le lancement des réformes Hartz sur le marché de l'emploi à partir de la fin 2003, ces inquiétudes se diffusent aussi dans les classes moyennes. Désormais, non seulement plus de la moitié des faibles revenus, mais également $43 \%$ des tranches moyennes de revenus se montrent pessimistes quant à l'avenir ; c'est ce qu'il ressort de la dernière édition du baromètre GMF réalisé par l'Institut IKG de l'Université de Bielefeld (Heitmeyer, 2008). La crainte d'être relégué au bas de l'échelle sociale atteint aujourd'hui largement les classes moyennes : elle affecte $50 \%$ des sondés aux revenus moyens. L'écart s'est nettement réduit avec les catégories à faibles revenus, qui sont $65 \%$ à redouter un déclassement, mais aussi avec les revenus supérieurs ; un tiers d'entre eux exprime la même crainte.

Les Allemands sont donc de plus en plus nombreux à se montrer insatisfaits des réalités économiques et à les considérer comme injustes. Un sondage réalisé par l'Institut für Demoskopie d'Allensbach pour le compte de diverses fondations, dont la Fondation Bertelsmann (Vehrkamp/Kleinsteuber, 2007) révèle qu'un Allemand sur huit seulement considère comme juste ou équitable la répartition des revenus et du patrimoine. Près des trois quarts de la population estime qu'elle est injuste ; en 2005, la moitié seulement était de cet avis, et un bon quart la considérait comme juste. Dix ans auparavant, en 1995, ces deux opinions se répartissaient encore à égalité.

C'est dans les catégories moyennes et supérieures de revenus que l'insatisfaction est la plus grande désormais, comme le montrent les dernières données de l'étude annuelle Sozio-Oekonomisches Panel (SOEP) menée par l'institut économique DIW (2008). La part des sondés qui pensent que leur salaire est injuste a augmenté de $26 \%$ dans les troisième, quatrième et cinquième déciles de l'échelle des revenus où, depuis 1992, elle est passée de $38 \%$ à $48 \%$, de même que dans la catégorie allant du $6^{\mathrm{e}}$ au $10^{\mathrm{e}}$ décile, où elle a crû de $56 \%$, passant de $20 \%$ à près de $32 \%$.

Reflet de ce malaise, la confiance des Allemands dans leur modèle économique - l'économie sociale de marché - tend à se réduire. Le sondage réalisé par l'Institut für Demoskopie d'Allensbach montre ainsi que les Allemands ne sont plus que $31 \%$ à penser du bien de l'économie sociale de marché. $38 \%$ d'entre eux la considèrent désormais sous un angle négatif. Si à l'évidence, cela vaut surtout pour les Allemands de l'est (une large moitié d'entre eux), pour la première fois, en Allemagne de l'ouest aussi, la part des opinions négatives (35\%) dépasse celle des opinions positives (34\%).

Parallèlement, les Allemands attendent plus des pouvoirs publics. La part de ceux qui réclament une intervention plus poussée de l'Etat dans la protection sociale est passée en deux ans de $43 \%$ à $60 \%$, ainsi que le révèle le sondage de l'institut ipos pour la Fédération des banques allemandes. Aujourd'hui, un quart seulement des Allemands souhaite plus de marché. Alors que ces dernières années, la part de ceux qui considèrent la garantie d'un bon niveau de protection et de confort social relève d'abord de la responsabilité de chacun ne cessait d'augmenter, on observe depuis 2006 une inversion de tendance. La part de ceux qui prônent l'apport individuel est tombée de $70 \%$ à $62 \%$ depuis. A l'opposé, la part de ceux qui considèrent qu'il revient à l'Etat d'œuvrer pour le bienêtre social est passée de $25 \%$ à $31 \%$ en deux ans

Les mutations du marché de l'emploi dans le fil de la globalisation des activités, la hausse continue du coût de l'énergie, ainsi que les licenciements massifs au mo- 
ment où les bénéfices des entreprises s'envolent et que les salaires des dirigeants atteignent des proportions vertigineuses, tous ces éléments ont contribué à semer un doute profond dans la population. C'est dans les tranches inférieures et moyennes des revenus qu'il est le plus prononcé, comme le révèle l'étude de l'institut IKG de Bielefeld: près des trois quarts des foyers à faibles revenus et près des deux tiers des foyers à revenus moyens disent avoir perdu toute orientation dans un monde qu'ils ne reconnaissent plus.

\section{La plus forte disparité des revenus est avant tout d'origine sociodémographique}

L'impression qu'ont les Allemands d'avoir perdu leurs repères, leur peur de subir un déclassement social ne se nourrissent pas seulement de leur situation économique et sociale individuelle. Elles sont entretenues bien plus encore par un ensemble de perceptions et d'appréciations négatives qui se diffusent dans l'opinion principalement via les médias. Or les présentations ainsi véhiculées sont à la limite de la caricature car elles reposent sur une vision partielle des faits et ne tiennent pas compte de l'ensemble des éléments à prendre en considération. Cela vaut tout particulièrement pour la présentation de l'évolution des revenus. La lecture indifférenciée qui en est faite dans l'espace public se concentre presque exclusivement sur deux catégories de revenus: ces classes moyennes qui fondent comme peau de chagrin, ainsi que ces faibles revenus dont la part s'est considérablement accrue, et où on observe une forte hausse du nombre d'enfants pauvres.

Il est vrai que, ces dernières années, l'écart s'est creusé au détriment des classes moyennes. L'étude SOEP fait apparaître que, entre 1996 et 2006, celles-ci ont 'perdu' 5,5 millions de personnes. Sur la même période, le nombre de personnes à faibles revenus a augmenté de 4,1 millions, dont 0,7 million d'enfants. Parallèlement, la tranche des hauts revenus a grossi elle aussi, bien qu'un peu moins, et compte désormais 2,1 millions de personnes de plus.

Evolution de la structure des revenus *) en Allemagne (1996-2006)

\begin{tabular}{|lcrr|}
\hline Revenus... & \% du revenu médian mensuel & $\mathbf{2 0 0 6}$ & $\mathbf{1 9 9 6}$ \\
... supérieurs & $>200 \%(>3125 €)$ & $9,2 \%$ & $6,4 \%$ \\
& $150-200 \%(>2344 €)$ & $11,3 \%$ & $11,6 \%$ \\
\hline ... moyens & $70-150 \%(>1094 €)$ & $54,1 \%$ & $61,3 \%$ \\
\hline$\ldots$ faibles & $60-70 \%(>938 €)$ & $7,2 \%$ & $7,5 \%$ \\
& $<60 \%$ (= seuil de pauvreté $:<938 €)$ & $18,3 \%$ & $13,2 \%$ \\
\hline
\end{tabular}

Source des données : IWG Bonn, Von Verlierern und Gewinnern, 2008. *) Revenu net disponible par unité de consommation, aux prix de 2008 = revenu du ménage rapporté au nombre de personnes ( 1 unité de consommation pour le premier adulte, 0,5 unité pour les personnes de plus de 14 ans, 0,3 pour les moins de 14 ans, selon l'échelle utilisée par l'OCDE, Eurostat, Destatis ou l'INSEE). NB : en 2006, le revenu médian mensuel était de $1563 €$ (aux prix de 2008).

\section{Structure sociodémographique de la population en RFA}

\begin{tabular}{|lcc|cc|rr|}
\hline & \multicolumn{2}{c|}{ \% dans la population } & \multicolumn{2}{c|}{$\begin{array}{c}\text { \% dans les revenus } \\
<\mathbf{7 0} \% \text { du médian }\end{array}$} & \multicolumn{2}{c|}{$\begin{array}{c}\text { \% dans les revenus } \\
>\mathbf{1 5 0} \% \text { du médian }\end{array}$} \\
& $\mathbf{2 0 0 6}$ & $\mathbf{1 9 9 6}$ & $\mathbf{2 0 0 6}$ & $\mathbf{1 9 9 6}$ & $\mathbf{2 0 0 6}$ & $\mathbf{1 9 9 6}$ \\
De souche allemande & 76,9 & 81,1 & 60,3 & 68,2 & 88,1 & 90,3 \\
Issue de I'immigration & 23,1 & 18,9 & 39,7 & 31,7 & 11,9 & 9,7 \\
$\mathbf{< 2 5}$ ans & 25,8 & 27,1 & 35,8 & 37,4 & 16,5 & 16,0 \\
$\mathbf{2 5 - 6 4}$ ans & 55,7 & 57,1 & 49,2 & 46,9 & 66,4 & 71,2 \\
> 65 ans & 18,5 & 15,8 & 14,9 & 15,6 & 17,0 & 12,9 \\
Célibataire & 18,4 & 16,6 & 22,0 & 20,4 & 16,3 & 14,8 \\
Couple sans enfants & 27,6 & 24,8 & 18,4 & 14,4 & 40,4 & 39,3 \\
Couple avec enfants & 44,2 & 47,6 & 41,7 & 46,0 & 40,2 & 37,7 \\
Foyer monoparental & 6,8 & 5,8 & 13,6 & 13,0 & 2,1 & 3,5 \\
Autre & 2,9 & 5,3 & 4,4 & 6,2 & 1,0 & 4,7 \\
\hline
\end{tabular}

Source des données : IWG Bonn, Von Verlierern und Gewinnern - Die Einkommensentwicklung ausgewählter Bevölkerungsgruppen in Deutschland, Bonn, juin 2008. 
.. principalement du fait des mutations démographiques

Immigration et éclatement des structures familiales gonflent la catégorie des bas revenus
L'analyse détaillée de ces données montre toutefois à quel point les conclusions qu'on en tire dans les virulents débats publics sont faussées : la fonte des classes moyennes ou leur déclassement dans l'échelle des revenus ne s'explique que partiellement par le chômage ou une baisse des salaires. En effet, il y a d'autres causes, plus fondamentales, en premier lieu desquelles les mutations qu'on observe dans la structure démographique de l'Allemagne.

Bien que la population allemande de souche se soit réduite de 2,8 millions d'individus entre 1996 et 2006, la population totale recensée en Allemagne s'est accrue de 0,7 million de personnes. Car durant cette période, la part des habitants issus de l'immigration a augmenté de 3,5 millions. Or quand on considère ces deux catégories de population sous l'angle de la structure de leurs revenus, on observe de fortes disparités. Ainsi, la part des Allemands de souche a diminué principalement dans les tranches moyennes de revenus alors que celle de la population issue de l'immigration a nettement augmenté dans les tranches les plus basses. La catégorie des faibles revenus s'est également accrue du fait de l'éclatement des structures familiales qui se traduit par un déclassement des foyers monoparentaux dans l'échelle des revenus. Enfin, dernier facteur d'explication de la fonte des classes moyennes: l'ascension d'un nombre important d'Allemands de souche vers les catégories supérieures de revenus.

\section{L'accroissement de la population à faibles revenus est imputable surtout aux immigrés et à leurs enfants, ainsi qu'aux familles monoparentales de souche allemande}

La hausse du nombre de foyers à faibles revenus est imputable aux trois quarts à l'immigration

Ces causes structurelles sont rarement prises en considération dans les débats publics outre-Rhin. II en va de même d'un autre fait déterminant pour comprendre la progression de la part des faibles revenus : l'insuffisante intégration de la population immigrée. En effet, en comparaison de la population de souche, on recense dans cette catégorie un plus faible taux d'activité, un plus fort taux de chômage, un faible niveau de qualification et une maîtrise lacunaire de la langue allemande, pour n'évoquer que les principaux traits structurels. Ainsi, sur les 3,5 millions de personnes qui sont venues entre 1996 et 2006 grossir les rangs de la population issue de l'immigration, plus de 2,9 millions (83\%) figurent en 2006 dans la catégorie des plus faibles revenus. Les $17 \%$ restants appartenaient tous à celle des revenus supérieurs. Pour le dire autrement: la hausse du nombre de foyers à faibles revenus recensés outre-Rhin (4,1 millions en 2006) est imputable à près des trois quarts à l'immigration.

Evolution de la structure des revenus dans la population issue de l'immigration en RFA ( 1996-2006)

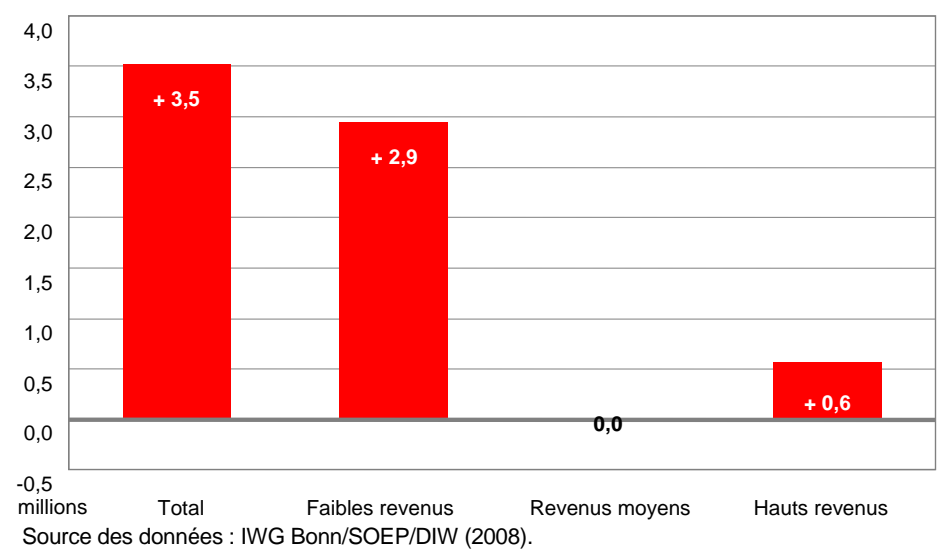

Si on considère les 2,9 millions d'immigrés à faibles revenus qui se sont installés en Allemagne entre 1996 et 2006 sous l'angle de la structure des foyers, on 
s'aperçoit que les couples avec enfants en constituent les deux tiers (2 millions de personnes). En un mot: ces familles immigrées additionnelles se situent presque en totalité dans la catégorie des faibles revenus.

Evolution de la situation des revenus des familles issues de l'immigration en RFA (1996-2006)

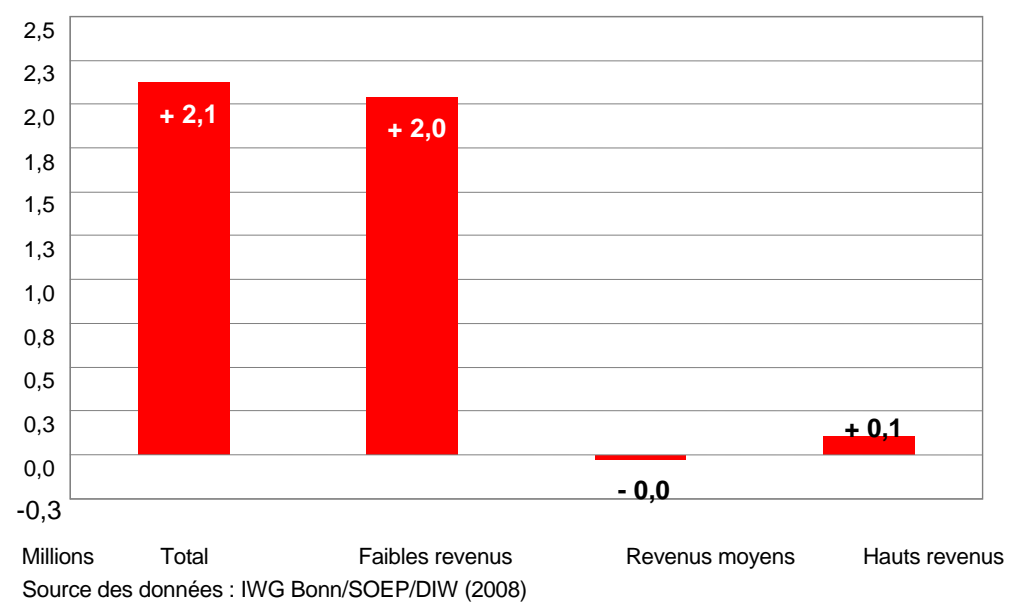

Cette tendance est plus prononcée encore chez les enfants de ces couples immigrés ; leur nombre a augmenté de 400000 en dix ans. Or près de 600000 enfants issus de l'immigration, soit nettement plus de $100 \%$ de ceux-là, sont venus grossir le groupe des bas revenus. 20000 seulement se classent dans les hauts revenus. Durant la même période, dans les revenus moyens, le nombre d'enfants issus de l'immigration s'est réduit de 200000.

Evolution des enfants d'immigrés sur l'échelle des revenus en RFA (1996-2006)

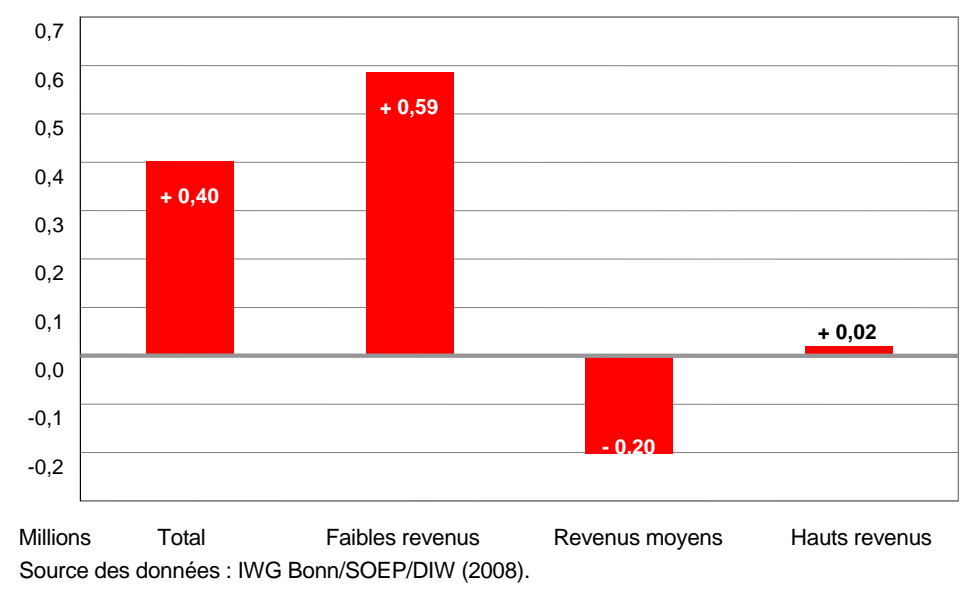

Cela signifie que la progression si souvent incriminée de la pauvreté chez les enfants est en réalité une conséquence directe d'une mauvaise intégration des enfants d'immigrés : la hausse du nombre d'enfants dans la catégorie des bas revenus les concerne à $82 \%$, et seulement à $18 \%$ les enfants de souche allemande.

Outre les immigrés et, dans ce groupe, les familles avec enfants, ce sont également les familles monoparentales de souche allemande qu'il convient de considérer comme les perdants dans l'évolution des revenus. Car souvent, la séparation des parents se traduit par le déclassement de tous les membres de la famille puisque la création de deux nouveaux foyers mène, à revenus généralement équivalents, à une hausse des dépenses pour le père et la mère. En 2006, plus d'un foyer monoparental sur deux appartient ainsi à la catégorie des faibles revenus. En dix ans, le nombre de familles monoparentales de souche allemande

... et leurs enfants

Faibles revenus : un nombre croissant de familles monoparentales 
s'est accru de $900000 ; 91 \%$ de ceux-ci (800 000 foyers) se situent dans les bas revenus.

Evolution des familles monoparentales de souche allemande sur l'échelle des revenus (1996-2006)

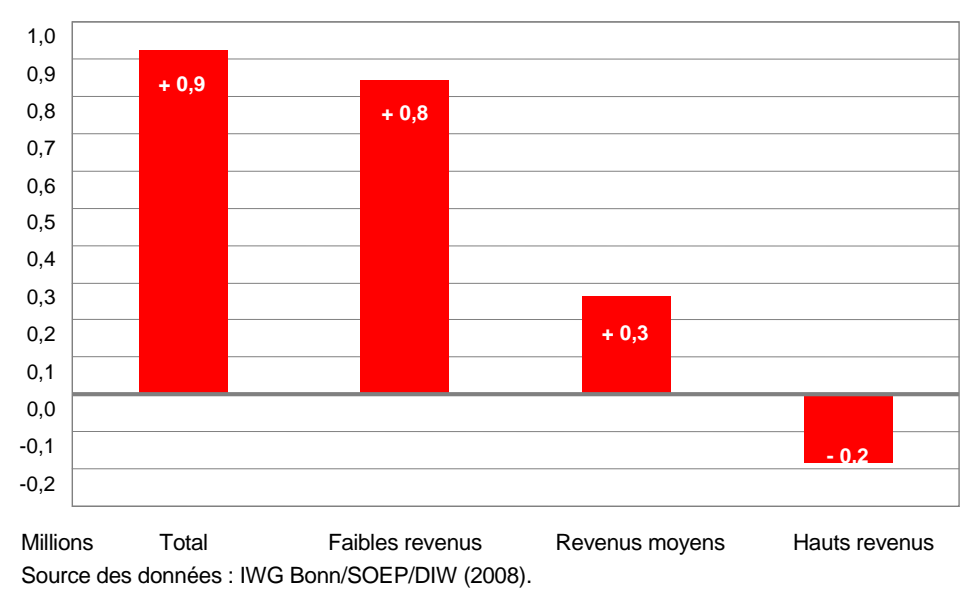

En d'autres termes : les familles monoparentales de souche allemande ont contribué à hauteur de $73 \%$ à grossir la part des foyers de souche allemande dans le segment des bas revenus (ils sont aujourd'hui 1,1 million).

\section{Gagnants dans l'évolution des revenus : les familles traditionnelles de souche allemande et les plus de 64 ans}

Contrairement à ce que laissent entendre les arguments exposés dans les débats publics, les familles traditionnelles avec enfants de souche allemande font partie des gagnants dans l'évolution des revenus.

Evolution des couples de souche allemande avec enfants sur l'échelle des revenus en RFA (1996-2006)

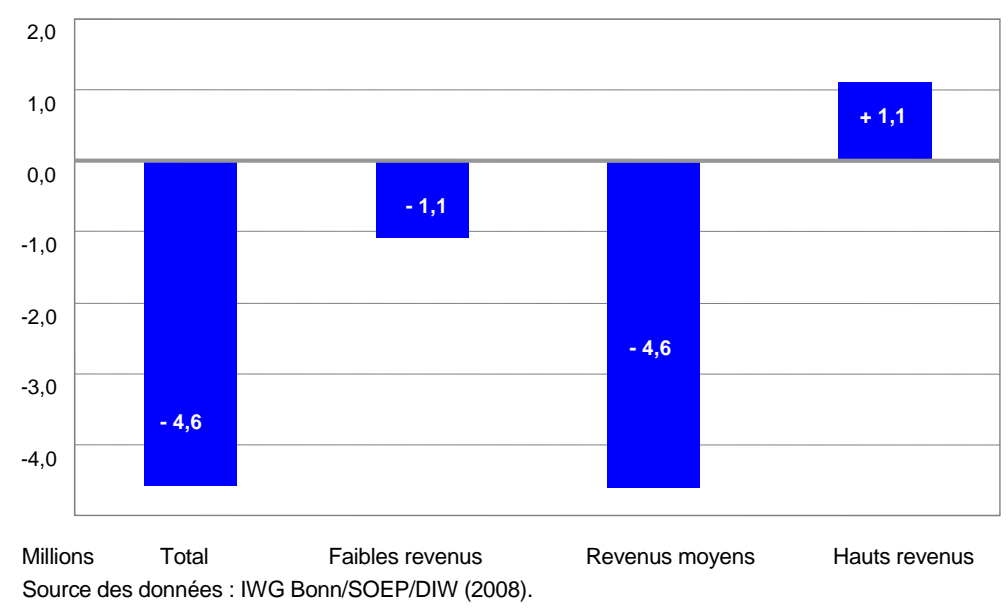

Les enfants des couples de souche allemande ont bénéficié de l'ascenseur social
Dans la population allemande, ce groupe s'est réduit depuis 1996, perdant 4,6 millions d'individus. Or si cette diminution s'est effectuée essentiellement dans la catégorie des revenus moyens, elle coïncide, du moins arithmétiquement, avec une hausse dans celle des revenus supérieurs. Ainsi, sur les 2,1 millions de personnes qui sont entrées dans cette dernière catégorie au cours de la décennie, $52 \%$ ( 1,1 million) sont des couples de souche allemande avec enfants. Autrement dit, à l'opposé des enfants d'immigrés, les enfants des couples de souche allemande ont, eux, bénéficié de l'ascenseur social. Et ce sont eux, selon toute vraisemblance, qui se partagent l'essentiel de ces quelque 23 milliards $€$ de reve- 
nus dont disposaient en 2007 les 6 à 19 ans, à en croire une étude de l'institut munichois Iconkids \& Youth.

Evolution des enfants des couples de souche allemande sur l'échelle des revenus en RFA (1996-2006)

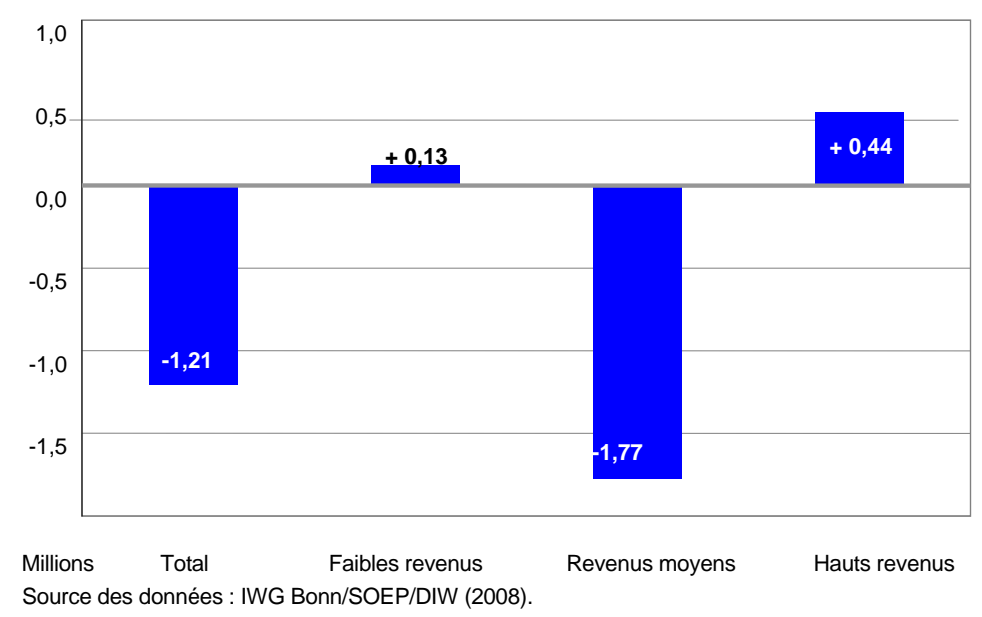

Parmi les gagnants figurent en outre incontestablement les plus de 64 ans. Les causes en sont principalement une plus large couverture des femmes par le régime légal des retraites, l'augmentation des retraites des salariés est-allemands après l'Unité, l'importance croissante des régimes privés de retraite comme des retraites d'entreprise, ainsi que la hausse du patrimoine financier et immobilier des plus âgés. Ainsi, sur cinq Allemands ayant franchi l'âge de 64 ans entre 1996 et 2006, deux entrent aujourd'hui dans la catégorie des revenus supérieurs, deux autres dans celle des revenus moyens et un seulement dans celle des faibles revenus.

Evolution des plus de 64 ans sur l'échelle des revenus en RFA (1996-2006)

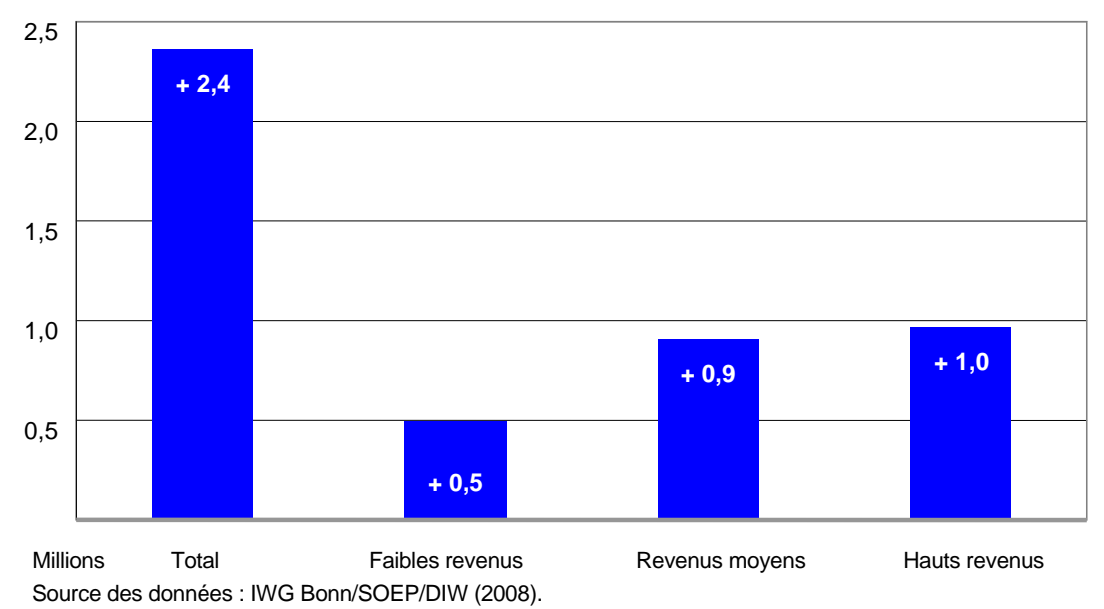

L'ENSEIGNEMENT A TIRER DE CES EVOLUTIONS STRUCTURELLES est simple. L'inégalité des revenus continuera de s'accroître aussi longtemps que, dans les milieux politiques comme dans l'espace public, ces évolutions seront considérées exclusivement comme un problème socio-économique et non pas aussi et avant tout comme un défi socio-démographique. Tant que les immigrés ne seront pas pleinement intégrés à la fois dans le marché du travail et dans le système éducatif, chaque vague d'immigration viendra inéluctablement grossir les rangs des faibles revenus. Et l'ascenseur social sera de plus en plus largement réservé aux Allemands de souche, une cohorte en baisse constante, ce qui gonflera la catégorie des hauts revenus et continuera à contracter les classes moyennes. 
L'évolution sociodémographique constitue un défi politique pour l'Allemagne. Pour contenir l'écartement de la fourchette des revenus et préserver le lien social, il convient avant tout de concevoir et de mettre en œuvre une politique démographique globale dont le pivot doit être une politique d'intégration assumée. II s'agit maintenant de réparer les erreurs du passé. Jusqu'à récemment, la mémoire de la sinistre pratique sous le régime national-socialiste nourrissait le rejet quasi unanime de toute politique démographique, y compris sous l'angle d'une politique d'immigration ciblée. Pour la même raison, on se refusait longtemps à considérer que ce qui, dans toute société, construit le lien social, c'est un niveau minimal d'identité culturelle et que, de ce fait, tout immigré est tenu d'apprendre la langue comme de se plier au droit et aux normes comportementales du pays d'accueil.

Cette nouvelle approche de la politique démographique doit avoir pour corollaire un effort accru en matière de qualifications, de soutien à la constitution d'un patrimoine, de même qu'elle doit s'accompagner d'un changement dans les mentalités et les comportements pour générer une approche plus respectueuse des intérêts de la collectivité. Car la multiplication des familles monoparentales accroît le nombre des personnes à faibles revenus. Aucune politique publique de transferts ne saura y remédier, tout au plus pourra-t-elle atténuer à la marge les effets de ces mutations. De plus, une telle politique impliquerait que la collectivité génère des moyens de plus en plus conséquents, mais ces moyens feraient alors défaut dans d'autres domaines eux aussi prioritaires.

Pour éviter une telle dérive et réduire en même temps l'inégalité des revenus, il n'y a qu'une voie : recréer l'équilibre entre les intérêts des particuliers, des familles et de la collectivité. Mais alors qu'en Allemagne, les milieux politiques comme l'opinion commencent à prendre plus clairement conscience du caractère incontournable d'une politique d'intégration affirmée, le changement de mentalité que requiert pourtant le défi sociodémographique est loin encore de s'esquisser, malgré quelques frémissements isolés.

Traduction : I. Bourgeois

\section{Indications bibliographiques}

ARD-Deutschlandtrend, 3 juillet 2008 (www.wdr.de)

BRÜCKER H., Ringer S., "Ausländer in Deutschland. Vergleichsweise schlecht qualifiziert », IAB-Kurzbericht, $\mathrm{n}^{\circ} 1 / 2008$

BUNDESVERBAND DEUTSCHER BANKEN, Wirtschaftsstandort 2008 -Ergebnisse einer repräsentativen Meinungsumfrage im Auftrag des Bundesverbandes deutscher Banken, Berlin, 2008

DESTATIS, Familienland Deutschland, Begleitmaterial zur Pressekonferenz, 22 juillet 2008 (www.destatis.de)

DESTATIS, « Jede vierte Familie in Deutschland hat einen Migrationshintergrund ", communiqué, 5 août 2008 (www.destatis.de)

GRABKA M. M., FRICK J., «Schrumpfende Mittelschicht - Anzeichen einer dauerhaften Polarisierung der verfügbaren Einkommen ? ", DIW-Wochenbericht, $n^{\circ} 10$, mars 2008

HeitmeYeR W., Hütting S., "Auf dem Weg in eine inhumane Gesellschaft - Die Abstiegsangst hat die Mittelschicht gepackt - mit gefährlichen Folgen für das soziale Klima ", Süddeutsche Zeitung, n`243, 21/22 octobre 2006

HeitMEYER W. (ed), Deutsche Zustände, vol. 1-6, Francfort/Main, 2002/08

"Kids : Immer mehr Geld, aber kaum mehr Ausgaben », 8 juillet 2008 (www.iconkids.de)

"Les niveaux de vie en 2006 », INSEE Première, juillet 2008 (www.insee.fr)

Miegel M., WAHL S., Schulte M., Von Verlierern und Gewinnern - Die Einkommensentwicklung ausgewählter Bevölkerungsgruppen in Deutschland, Institut für Wirtschaft und Gesellschaft Bonn, Bonn, juin 2008

Moati P., Rochefort R., Mesurer le pouvoir d'achat, Conseil d'analyse économique, rapport, 2008 (www.cae.gouv.fr)

SOEP/DIW, Sozio-Oekonomisches Panel/Deutsches Institut für Wirtschaftsforschung, Sonderauswertung für das IWG BONN, Berlin, 2008

VehrKAmp R. B., KLEINSTEUBer A., Soziale Gerechtigkeit 2007 - Ergebnisse einer repräsentativen Bürgerumfrage, Gemeinschaftsinitiative der Bertelsmann Stiftung, Heinz Nixdorf Stiftung und Ludwig-Erhard-Stiftung, Gütersloh, 2007

«25 Wellen Sozio-oekonomisches Panel », DIW-Vierteljahrshefte zur Wirtschaftsforschung, $n^{\circ} 3 / 2008$ 